\title{
Position Tracking of a Pneumatic-Muscle-Driven Rehabilitation Robot by a Single Neuron Tuned PID Controller
}

\author{
Jun Zhong $\mathbb{D}^{1}{ }^{1}$ Yue Zhu, ${ }^{1}$ Chun Zhao, ${ }^{1}$ Zhenfeng Han, ${ }^{2}$ and Xin Zhang ${ }^{3}$ \\ ${ }^{1}$ College of Mechanical \& Electrical Engineering, Hohai University, Changzhou 213022, Jiangsu Province, China \\ ${ }^{2}$ HRG Institute (Hefei) of International Innovation, Hefei 230000, Anhui Province, China \\ ${ }^{3}$ Technology Center, Xinxing Cathay International Group, Beijing 100070, China \\ Correspondence should be addressed to Jun Zhong; zhongjun@hhu.edu.cn
}

Received 9 July 2020; Revised 9 September 2020; Accepted 26 September 2020; Published 8 October 2020

Academic Editor: Yanan Li

Copyright (c) 2020 Jun Zhong et al. This is an open access article distributed under the Creative Commons Attribution License, which permits unrestricted use, distribution, and reproduction in any medium, provided the original work is properly cited.

Pneumatic muscle actuators (PMAs) own compliant characteristics and are suitable for use in rehabilitation equipment. This paper introduces a rehabilitation robot driven by PMAs devised in the Rehabilitation and Medical Robot Laboratory. Considering high nonlinearities inside PMAs, a single neuron tuned PID controller is carefully designed. Experimental setup is built up and trials are performed. Results demonstrate the proposed advanced PID algorithm can achieve better capacity in position tracking than the conventional PID controller.

\section{Introduction}

Features of physiological function recession in aging process include decreased limb flexibility, osteoporosis, muscle atrophy, and significant decline in loading capacity of bone tissue. The incidence of acute cardiovascular and cerebrovascular diseases and that of neurological diseases in the aged keep at a high level, and most of these patients have symptoms of hemiplegia $[1,2]$. Quantity of patients with limb dyskinesia caused by other diseases, sports injury, and traffic accidents is increasing rapidly. For these patients, besides early surgical treatments and necessary medications, correct and scientific rehabilitation training plays an important role in restoration and improvement of limb motion functions [3]. Many patients suffer from muscle atrophy and lose limb mobility because of improper training methods and failure to get effective rehabilitation training. This unfortunately makes patients suffer tremendously and causes great burden to family and society $[4,5]$. A lot of clinical practices have proved that, without scientific and sufficient rehabilitation training, many patients with impairment of limb motion functions cannot restore the walking capacity to normality and have to move in a typical asymmetric attitude. Thus, improvements in patients' walking capacity and the ability to take care of themselves in lives make a lot of sense to both patients and society. However, dependence only on physiotherapists cannot meet requirements of patients' rehabilitation because the amount of physiotherapists is small and one physiotherapist lacks the capacity of training several patients simultaneously. Another barrier is that different physiotherapists have different understandings about rehabilitation exercises and different evaluating indicators [6-10]. The above obstacles are harmful in achieving ideal training strength and effects. Besides, loss of necessary means of detection and control makes obstacles in meeting the needs of a large number of patients in modern society. Rehabilitation robots can help patients complete kinds of motion function rehabilitation training, such as arm restoring therapy and ankle rehabilitation [11-13]. Wu et al. designed a three-degrees-of-freedom lower rehabilitation robot involving hip, knee, and ankle joints and proposed an adaptive robust subcontroller for the robot to handle system uncertainties and disturbances from patients [14] Banala et al. developed a robot-assisted gait training algorithm and used a force-field controller to achieve more effective training [15]. Besides, other rehabilitation robots have been developed [16-29]. Fateh and Khoshdel presented a new voltage-based adaptive impedance force control for a lower 
limb rehabilitation robot, and gradient descent algorithm was adopted to tune impedance parameters to guarantee force controlling effect [30].

Most of the presented rehabilitation robots adopted motors as actuators, which leads the lack of compliance during recovery. In this research, a compliant ankle-rehabilitation robot driven by PMAs is presented and an advanced PID algorithm is devised to handle nonlinearities and disturbances inside the robot. This paper is arranged as follows. Section 2 introduces characteristics of the devised pneumatic muscle actuator-driven ankle rehabilitation robot. Section 3 proposes a single neuron adaptive PID controller for the robot; Section 4 establishes the experimental setup of the robot and performs the rehabilitation tests to validate the designed controller.

\section{Structure of the Ankle-Rehabilitation Robot}

2.1. Structure Description. The detailed structure of the ankle rehabilitation robot is addressed prudently and shown in Figure 1. Decomposition diagram of the ankle joint mechanism is displayed in Figure 2. One of the crucial issues to be handled is that placing angle and height of the training device must own the capacity of adjustment according to different patients and circumstances. Thus, the robot framework is made of aluminum profiles whose installing grooves are in unique T-shape (shown in Figure 3). By this way, arrangement angle and height of the robot can be adjusted arbitrarily in the framework. Besides, robot mounting panel has slots and holes. Slots allow the robot to move along and be fixed by bolts. Holes help the robot rotate around to modulate the arrangement angles and guarantee comfort during rehabilitation exercises. The panel framework is made up of aluminum profiles, whilst the robot is fabricated by light and high tensile aluminum. Another important issue is to guarantee safety during training exercises. This is realized by the limitation of the allowable angle range of the executive mechanism. Actually, angle limiting device (shown in Figure 4) is devised in the robot to avoid hurt on the patients' ankles. The range of the executive mechanism in the robot is $\left(-30^{\circ}, 30^{\circ}\right)$.

In traditional ankle rehabilitation robots, driving components are usually installed on one side of the ankle joint. This type of unilateral asymmetric structure (shown in Figure 5(a)) may exert uncertain force to joint, produces harmful torque on the robot joint in extra directions, and has detrimental influence on mechanical structure stability. Bilateral structure is adopted in the ankle joint design in order to preventing from producing harmful torques in extra directions. This bilateral structure keeps balance in different components of ankle rehabilitation robot and eliminates harmful torques created by drive power and forces from the feet of patients. Power supplying mechanism and transmission mechanism are both installed bilaterally about the ankle joint, which makes identical forces on the two sides of the structure. The symmetrical configuration can further guarantee the stability and reliability of mechanical transmission, make good use of space, and improve compact layout of the robot (shown in Figure 5(b)).

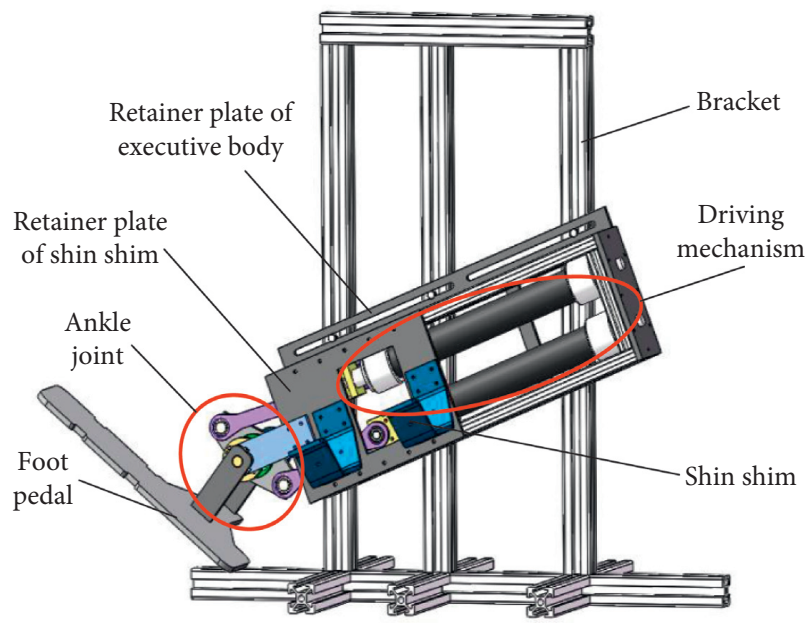

Figure 1: CAD model of the ankle rehabilitation robot.

2.2. Prototype. The prototype of the robot (shown in Figure 6) adopts aluminum alloy to make sure of light weight and high strength. Aluminum alloy profile frame is used to regulate posture of the robot for various patients. A torque sensor is installed in one side of the output shaft of the transmission mechanism to measure driving torque or the human ankle, and an incremental encoder is fixed with the other side of the identical output shaft to record the real time rotation angle.

\section{Single Neuron Tuned Adaptive PID Controller}

3.1. Single Neuron Adaptive PID Algorithm. Proportional Integral Differential (i.e., PID) algorithm is widely used in various rehabilitation and medical robots because of concision, efficiency, and reliability. However, high nonlinearity and strong hysteresis of PMAs bring a tricky job in keeping high tracking capacity because of which conventional PID algorithm owns constant values of parameters $P, I$, and $D$. Considering single neuron strategy has the excellent capacity in regulating structural parameters, a single neuron tuned PID controller is devised, as shown in Figure 7. $\omega_{1}, \omega_{2}$, and $\omega_{3}$ are structural parameters of single neuron algorithm and represent $P, I$, and $D$ of the PID controller, respectively. Values of $\omega_{1}, \omega_{2}$, and $\omega_{3}$ are online regulated according to some criterions, and this paper adopts a hybrid of supervisory delta learning rule and nonsupervisory Hebbian rule. A cost function is defined as the evaluation indicator of the tuning algorithm in the following equation:

$$
\Theta(k)=\frac{1}{2}\left[\theta_{\text {ref }}(k)-\theta(k)\right]^{2} .
$$

The principle is to reduce $\Theta(k)$ by online tuning parameters $\omega_{1}, \omega_{2}$, and $\omega_{3}$. Gradient descent algorithm is used as follows:

$$
\omega_{i}(k+1)=\omega_{i}(k)-\eta_{i} \frac{\partial \Theta(k)}{\partial \omega_{i}(k)}, \quad i=1,2,3 .
$$




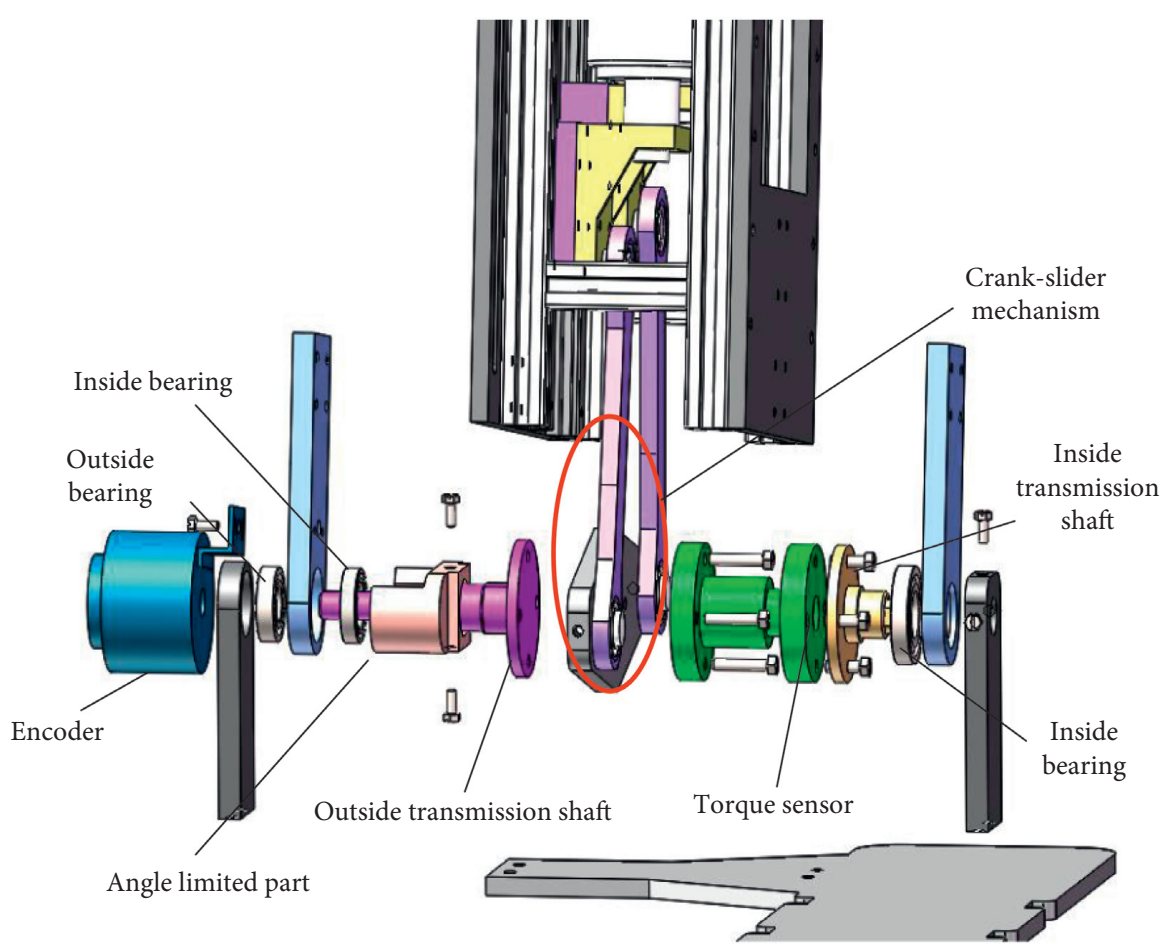

Figure 2: Decomposition diagram of the ankle joint mechanism.

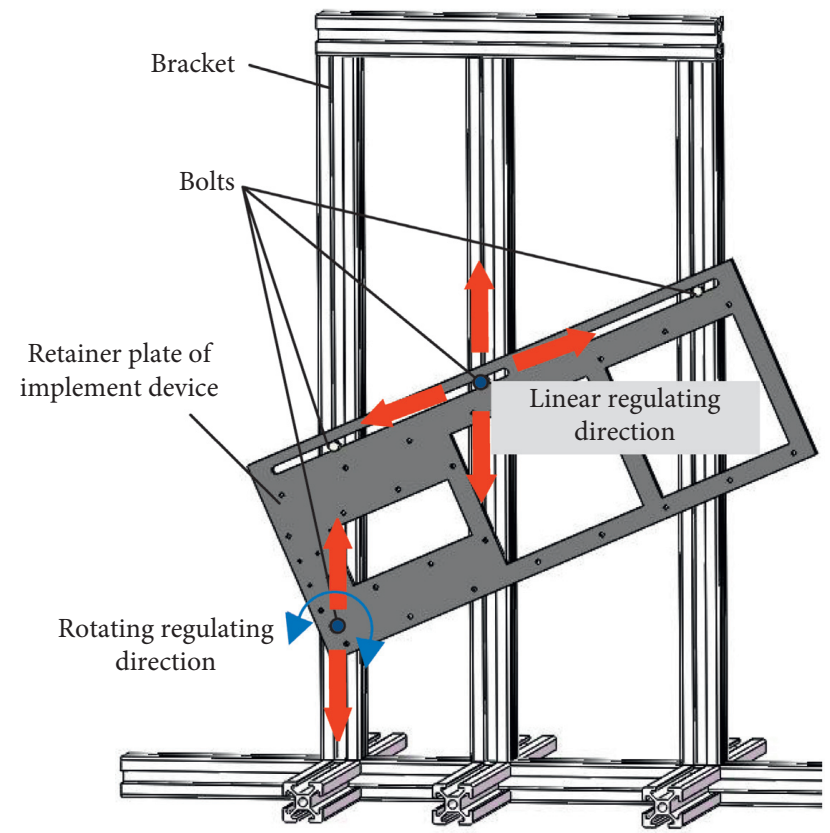

FIgURE 3: Regulating device for installing angle and height of the robot.

According to chain rule of differential equations, $\partial \Theta / \partial \omega_{i}(k)$ is calculated as follows:

$$
\frac{\partial \Theta}{\partial \omega_{i}}=\frac{\partial \Theta}{\partial \theta} \frac{\partial \theta}{\partial u_{\mathrm{pid}}} \frac{\partial u_{\mathrm{pid}}}{\partial \omega_{i}}=-e(k) \cdot \frac{\partial \Theta}{\partial u_{\mathrm{pid}}} \cdot x_{i} .
$$

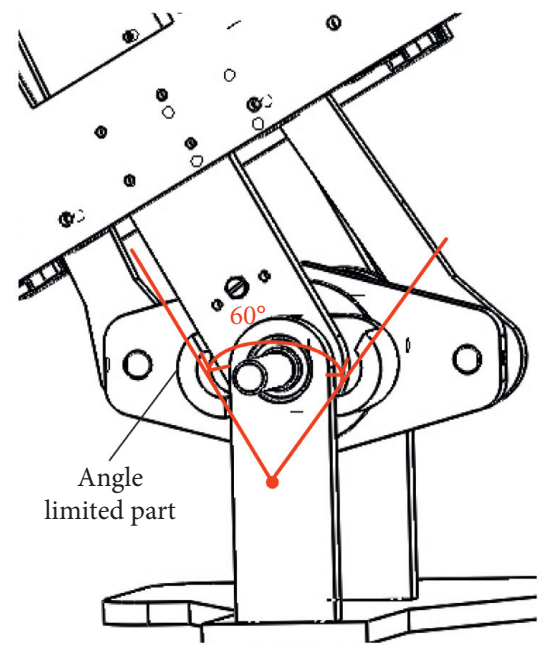

FIgure 4: Angle limited part.

Usually, $\partial \Theta / \partial u_{\text {pid }}$ is difficult to calculate because of complexity of the actual system. To simplify the calculation, it is replaced by $\operatorname{sgn}\left[\partial \theta / \partial u_{\text {pid }}\right] \cdot x_{i}(i=1,2,3)$ are inputs of neuron units $\omega_{1}, \omega_{2}$, and $\omega_{3}$, respectively. In the single neuron PID controller, $x_{i}$ are assigned according to tracking errors of the system, i.e.,

$$
\begin{aligned}
& x_{1}=e(k), \\
& x_{2}=e(k)-e(k-1), \\
& x_{3}=e(k)-2 e(k-1)+e(k-2) .
\end{aligned}
$$




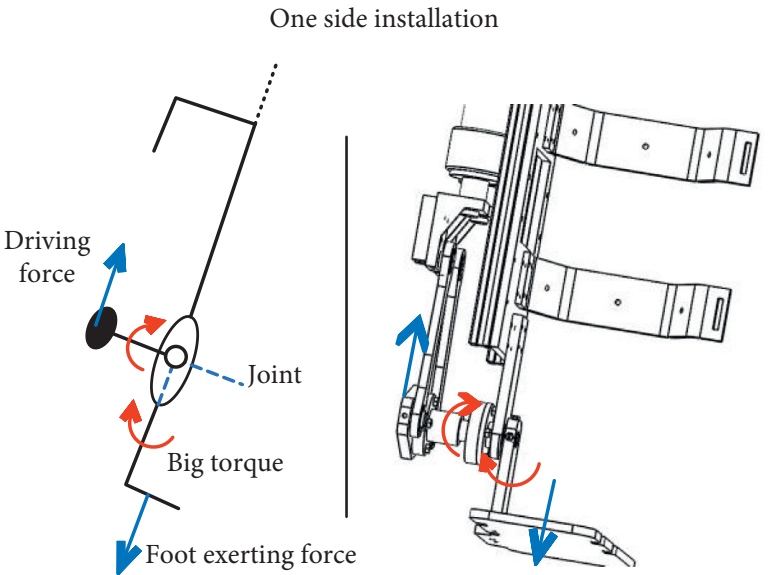

(a)

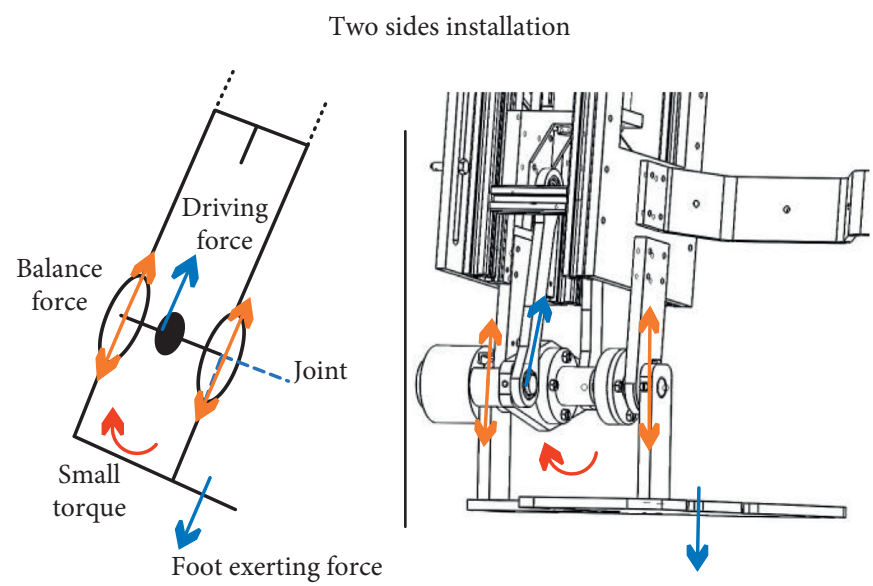

(b)

Figure 5: Comparison of two installing types of driving mechanism: (a) unilateral asymmetric structure; (b) symmetrical configuration.

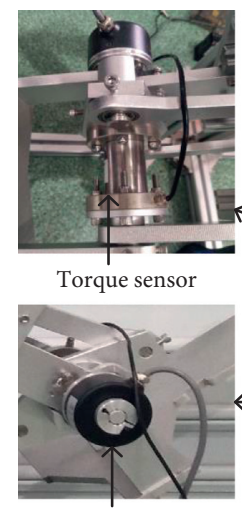

Incremental encoder

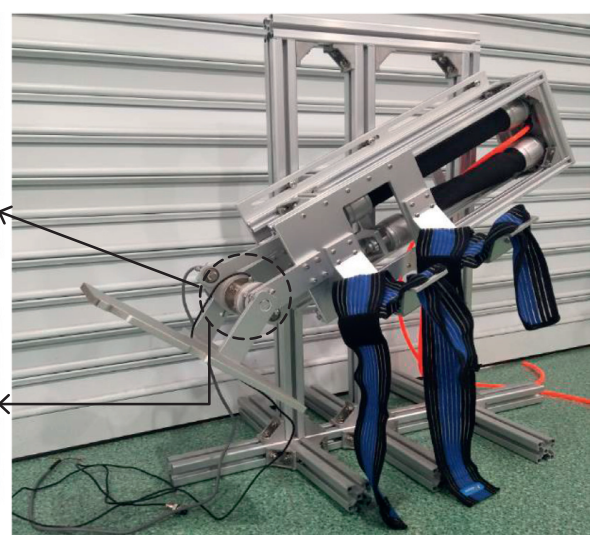

Figure 6: Prototype of the rehabilitation ankle robot driven by PMAs.

The output $u_{\text {pid }}$ is as follows:

$$
u_{\mathrm{pid}}=\frac{K \sum_{i=1}^{3}\left|x_{i} \omega_{i}\right|}{\sum_{i=1}^{3}\left|\omega_{i}\right|}
$$

3.2. Controller Design of the Rehabilitation Robot. The controller is devised using single neuron strategy tuned PID algorithm in Figure 7. Two electrical proportional valves with type of ITV1050-212N made by SMC Corporation are used in the setup to control the pressure inside the pair of PMAs. This type valve has an output range of pressure in $(0.005,0.9) \mathrm{MPa}$ and linear correspondent controlling voltage in $(0,5) V . u_{01}$ in Figure 8 is the initial input voltage of the first valve, i.e., the initial pressure inside the first PMA. Similarly, $u_{02}$ is the initial input voltage of the second valve, i.e., the initial pressure inside the second PMA. Values of $u_{01}$ and $u_{02}$ are tuned by the trial and error. $P_{\text {valve }}(u)$ is the transfer function of the proportional valve and has the following form:

$$
P_{\text {valve }}(u)=0.179 u+0.005 \text {. }
$$

Torque switch in the controlling block is used to guarantee the safety of patients by setting a range of torque. If the sampled torque value is larger than the upper limit $T_{\max }$, or less than lower limit $T_{\min }$, proportional valves will output zero to PMAs and the robot stops working. $T_{\max }$ and $T_{\min }$ are acquired by trial and error.

3.3. Stability Analysis of the Proposed Controller. Controlling parameters of single neuron PID algorithm vary along with the direction of negative gradient descent of $\omega_{1}$, $\omega_{2}$, and $\omega_{3}$. Stability of the single neuron PID controller is analyzed by Lyapunov principle. Firstly, a Lyapunov function is defined as follows:

$$
\mathbf{E}(\mathbf{k})=\Theta(k)=\frac{1}{2} e^{2}(k) .
$$

Variation of $\mathbf{E}(\mathbf{k})$ in the self-learning process of the single neuron model is expressed:

$$
\Delta \mathbf{E}(\mathbf{k})=\frac{1}{2} e^{2}(k+1)-\frac{1}{2} e^{2}(k) .
$$

Similarly, variation of $\mathbf{e}$ can be acquired as follows:

$$
e(k+1)=e(k)+\sum_{i=1}^{3} \frac{\partial e(k)}{\partial \omega_{i}(k)} \Delta \omega_{i}(k) .
$$

Considering

$$
\Delta \omega_{i}(k)=-\eta_{i} \frac{\partial \Theta(k)}{\partial e(k)} \frac{\partial e(k)}{\partial \omega_{i}(k)}=-\eta_{i} e(k) \frac{\partial e(k)}{\partial \omega_{i}(k)},
$$

then

$$
\Delta e(k)=e \sum_{i=1}^{3} \frac{\partial e(k)}{\partial \omega_{i}(k)} \eta_{i} \Delta \omega_{i}(k)=-e \widehat{O}^{T} \eta \widehat{O}
$$

where $\widehat{\mathrm{O}}=\left[\partial e(k) \partial \omega_{1}(k) \partial e(k) \partial \omega_{2}(k) \partial e(k) \partial \omega_{3}(k)\right]^{T}$. 


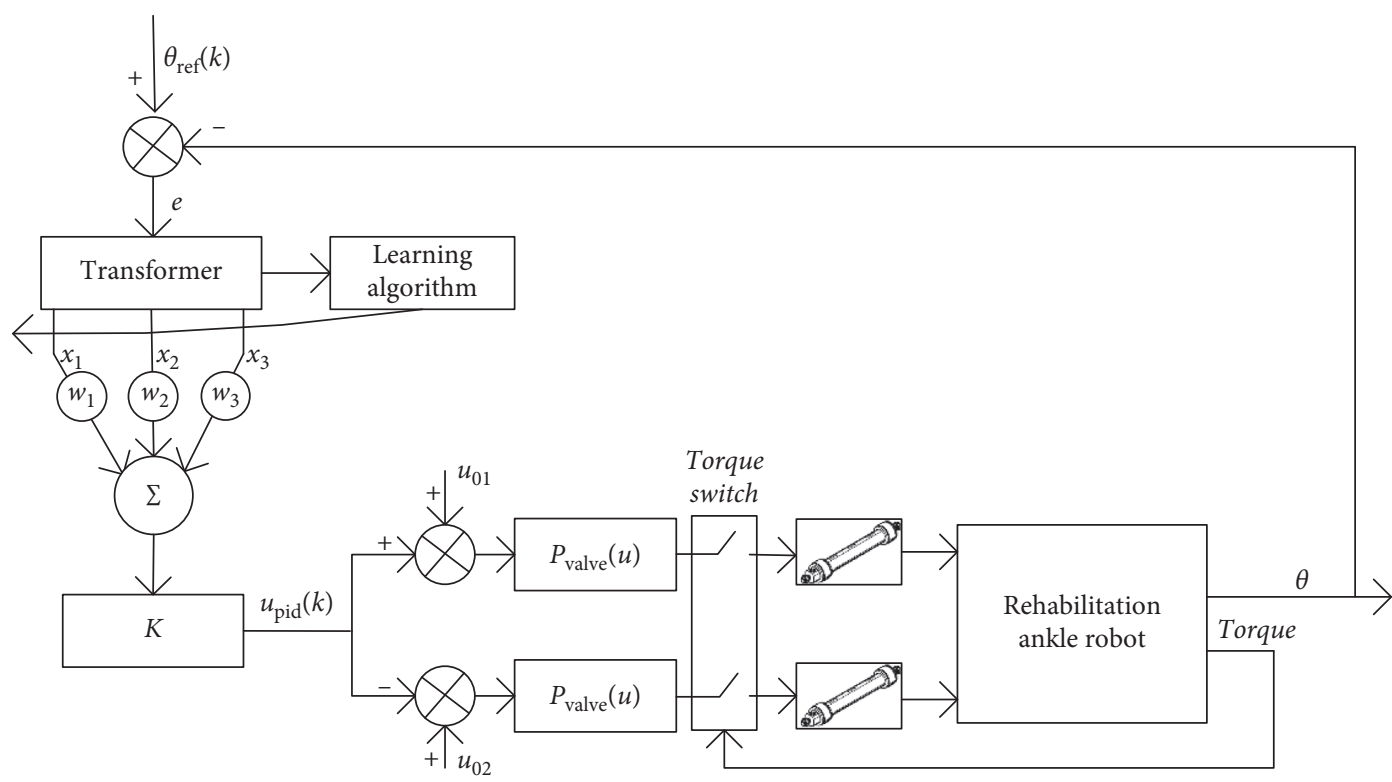

FIgURE 7: Tracking controller of the robot in passive rehabilitation therapy.

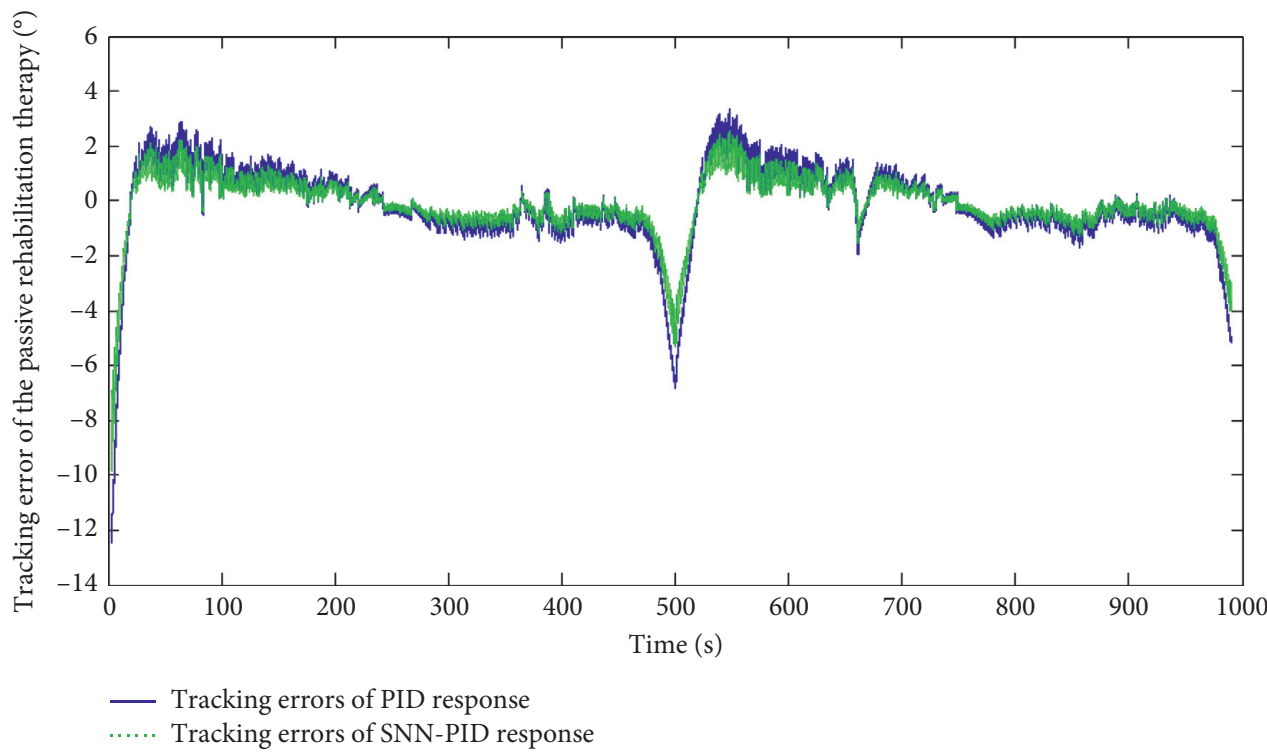

FIGURE 8: Tracking errors of passive rehabilitation therapy experiment under a half sinusoidal wave excitation of $0.002 \mathrm{~Hz}$ with amplitude $50^{\circ}$.

Thus,

$$
\Delta \mathbf{E}(\mathbf{k})=-\frac{1}{2}(e(k) \widehat{O})^{T}\left[2 \boldsymbol{\eta}-\mathbf{\eta} \widehat{O}^{\mathbf{T}}{ }^{\mathrm{T}} \eta\right](e(k) \widehat{O}) .
$$

Several conclusions can be acquired by Lyapunov principle:

(a) $\mathbf{E}(\mathbf{k})$ is positive definite

(b) If and only if $2 \eta-\eta \hat{O} \widehat{O}^{\mathrm{T}} \eta>0, \Delta \mathbf{E}(\mathbf{k})$ is negative definite, which means stability of the system depends on the learning step

(c) When $k$ approaches infinity, $\mathbf{E}(\mathbf{k})$ approaches zero

Obviously, proper values of $\eta$ make $\Delta \mathbf{E}(\mathbf{k})<0$, which means the controlling system keeps stable.

\section{Experimental Validations and Discussion}

The entire robot system, including controlling component and air compressor, is shown in Figure 9. An AD/DA card of USB3102A type from Beijing Art Technology Development Co., Ltd., is adopted to control the robot and samples all status information from sensors. An air compressor is employed as the compressed air supplier. Main components in the robot are listed in Table 1.

To validate the effectiveness of the proposed single neuron tuned PID algorithm, several trials are conducted under different excitation. Experiments are performed on a male with weight of $77 \mathrm{~kg}$ and height of $176 \mathrm{~mm}$ in this study (shown in Figure 10). It is generally known that passive rehabilitation therapy is used for serious dyskinesia of limbs and must operate 


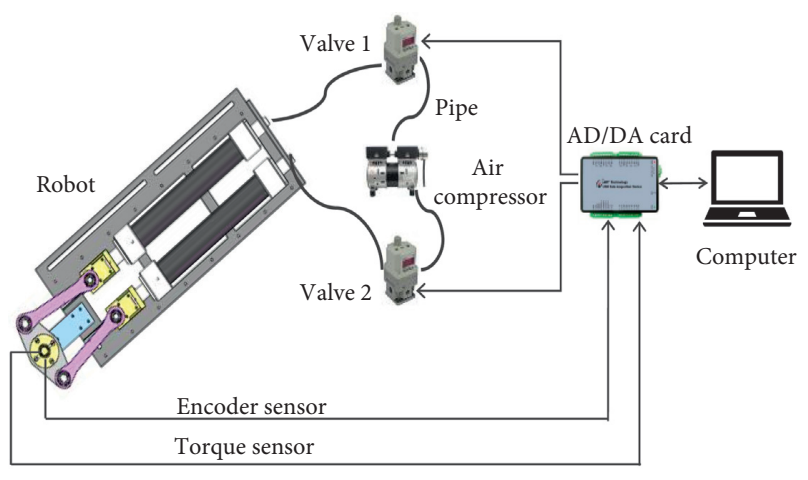

(a)

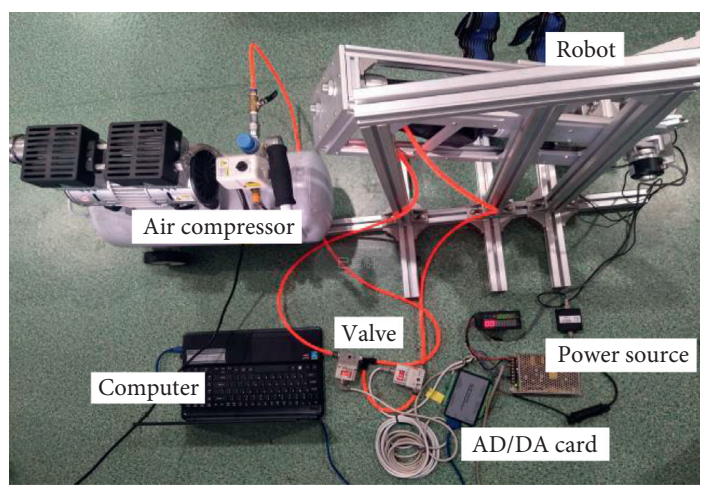

(b)

FIgURE 9: The PMAs driven rehabilitation ankle robot: (a) schematic drawing; (b) prototype of the experimental setup.

TABle 1: Components of the PMA-driven rehabilitation ankle robot.

\begin{tabular}{|c|c|c|c|}
\hline Name & Type & Manufacturer & Main parameters \\
\hline PMA & DMSP-40-300N-RMCM & FESTO corporation & $\begin{array}{l}\text { Nominal inner diameter: } 40 \mathrm{~mm} \\
\text { Nominal length: } 300 \mathrm{~mm} \\
\text { Pressure range: } 0-0.6 \mathrm{MPa} \\
\text { Maximum force: } 6000 \mathrm{~N} \\
\text { Maximum contractile ratio: } 25 \%\end{array}$ \\
\hline Valve & ITV1050-212N & SMC corporation & $\begin{array}{l}\text { Controlling voltage range: } 0-5 \mathrm{~V} \\
\text { Output pressure range: } 0.005-0.9 \mathrm{MPa}\end{array}$ \\
\hline Incremental encoder & ZMK60 & & $\begin{array}{c}\text { Working voltage: DC } 5 \mathrm{~V} \\
\text { Maximum frequency response: } 300 \mathrm{kHz}\end{array}$ \\
\hline Torque sensor & ZNNT-F & CHINO SENSOR & $\begin{array}{c}\text { Load range: } 2-200 \mathrm{Nm} \\
\text { Output signal: } 0-5 \mathrm{~V}, 0-10 \mathrm{~V}, 4-20 \mathrm{~mA}, 0-10 \mathrm{~mA} \\
\text { Comprehensive accuracy: } 0.2 \%\end{array}$ \\
\hline $\mathrm{AD} / \mathrm{DA}$ card & USB3102A & ART technology corporation & $\begin{array}{c}\text { AD module: } \\
\text { Resolution: } 16 \text { bit } \\
\text { Maximum sampling speed: } 250 \mathrm{Ksps} \\
\text { Range: } \pm 10 \mathrm{~V}, \pm 5 \mathrm{~V}, \pm 2 \mathrm{~V}, \pm 1 \mathrm{~V} \\
\text { DA module: } \\
\text { Resolution: } 16 \text { bit } \\
\text { Maximum sampling speed: } 100 \mathrm{Ksps}\end{array}$ \\
\hline Computer & INS14-3476 & DELL corporation & $\begin{array}{l}\text { Processor: Intel i5-7200U } \\
\text { Storage disk: } 500 \mathrm{G} \\
\text { RAM: } 4 \mathrm{~GB}\end{array}$ \\
\hline Air compressor & DET750-30L & DAERTUO corporation & $\begin{array}{c}\text { Work power: } 750 \mathrm{~W} \\
\text { Exhaust air capacity: } 120 \mathrm{~L} / \mathrm{min} \\
\text { Maximum pressure: } 0.8 \mathrm{MPa}\end{array}$ \\
\hline
\end{tabular}

at a very low speed. A half sinusoidal wave excitation of $0.004 \mathrm{~Hz}$ with amplitude $50^{\circ}$ is applied to the robot, and tracking responses and errors are plotted in Figures 11 and 12, respectively. Relative large errors occur at the zero position due to assembly errors of the robot and creep and high nonlinearities inside PMA. Figure 11(a) shows that the maximum tracking error of the PID controller is $5.905^{\circ}$ at the time of 548.3 $\mathrm{s}$, whilst that of single neuron tuned PID is $4.541^{\circ}$. Figure 11 (b) shows an enlarged part for the portion A in Figure 11(a), from where the conclusion that single neuron tuned PID controller achieves smaller tracking errors than conventional PID controller can be drawn. Figure 12 shows tracking errors of passive rehabilitation therapy in Figure 11, which further proves the better capacity of single neuron regulated PID than classic PID. Figure 13 displays a half sinusoidal wave excitation of $0.002 \mathrm{~Hz}$ with amplitude $50^{\circ}$ and different responses of the adaptive PID controller and classic PID controller. Figure 13(a) shows a maximum tracking error of $3.364^{\circ}$ from PID controller responses and a maximum tracking error of $2.603^{\circ}$. Figure $13(\mathrm{~b})$ shows an enlarged part for the portion A in Figure 13(a), which further proves that the single neuron tuned PID controller achieves smaller tracking errors than the conventional PID controller. Figure 8 shows the corresponding tracking error performances in Figure 13, and comparison between response 


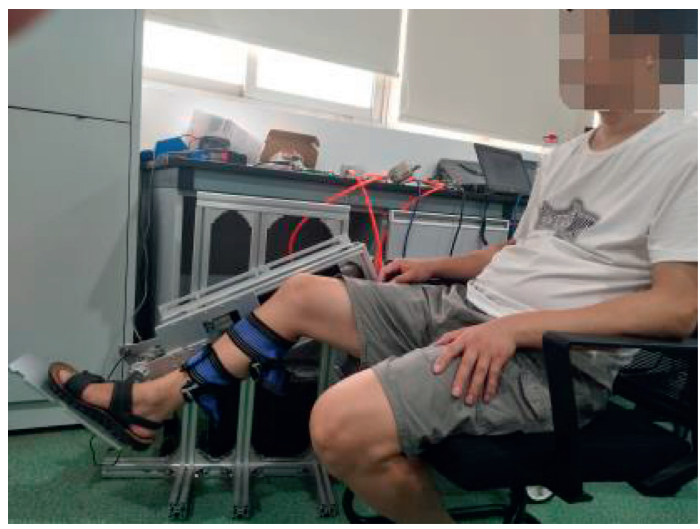

Figure 10: Experiment validations on a male with weight of $77 \mathrm{~kg}$ and height of $176 \mathrm{~mm}$.

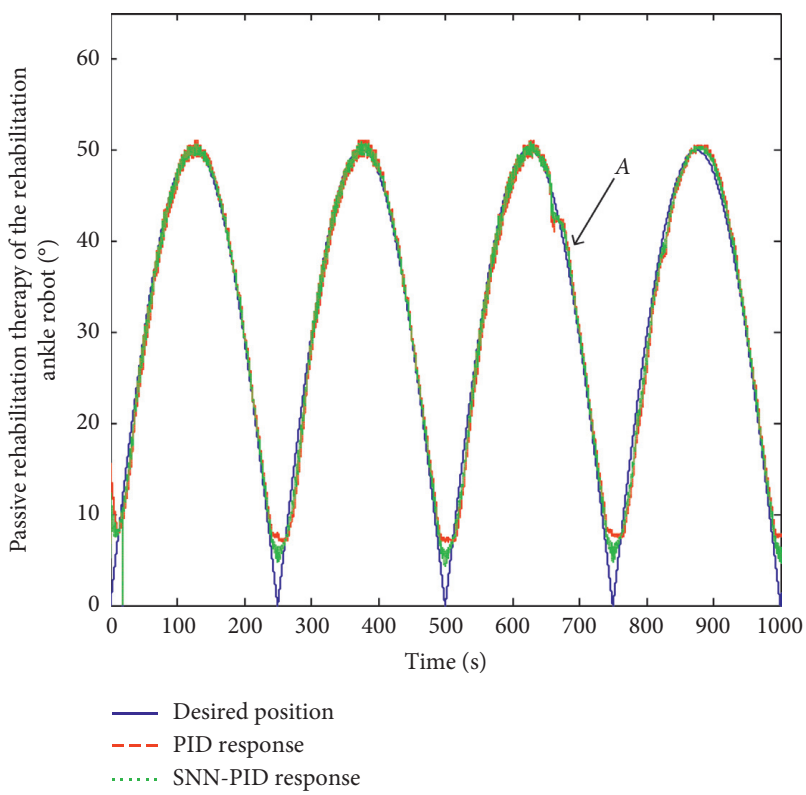

(a)

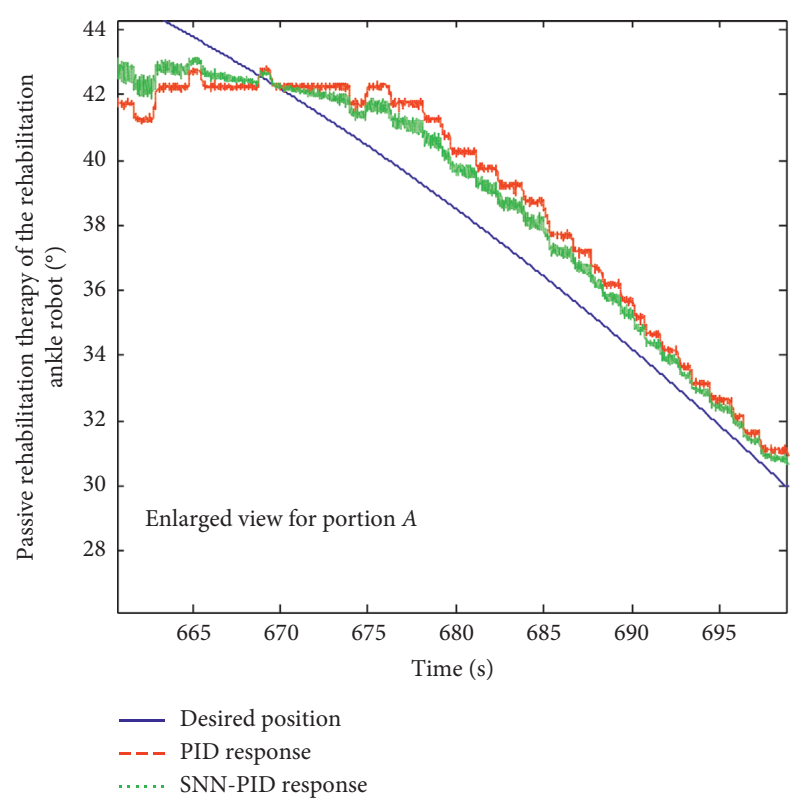

(b)

Figure 11: Passive rehabilitation therapy experiment under a half sinusoidal wave excitation of $0.004 \mathrm{~Hz}$ with amplitude $50^{\circ}$ : (a) tracking performance; (b) enlarged view for portion $\mathrm{A}$ in (a).

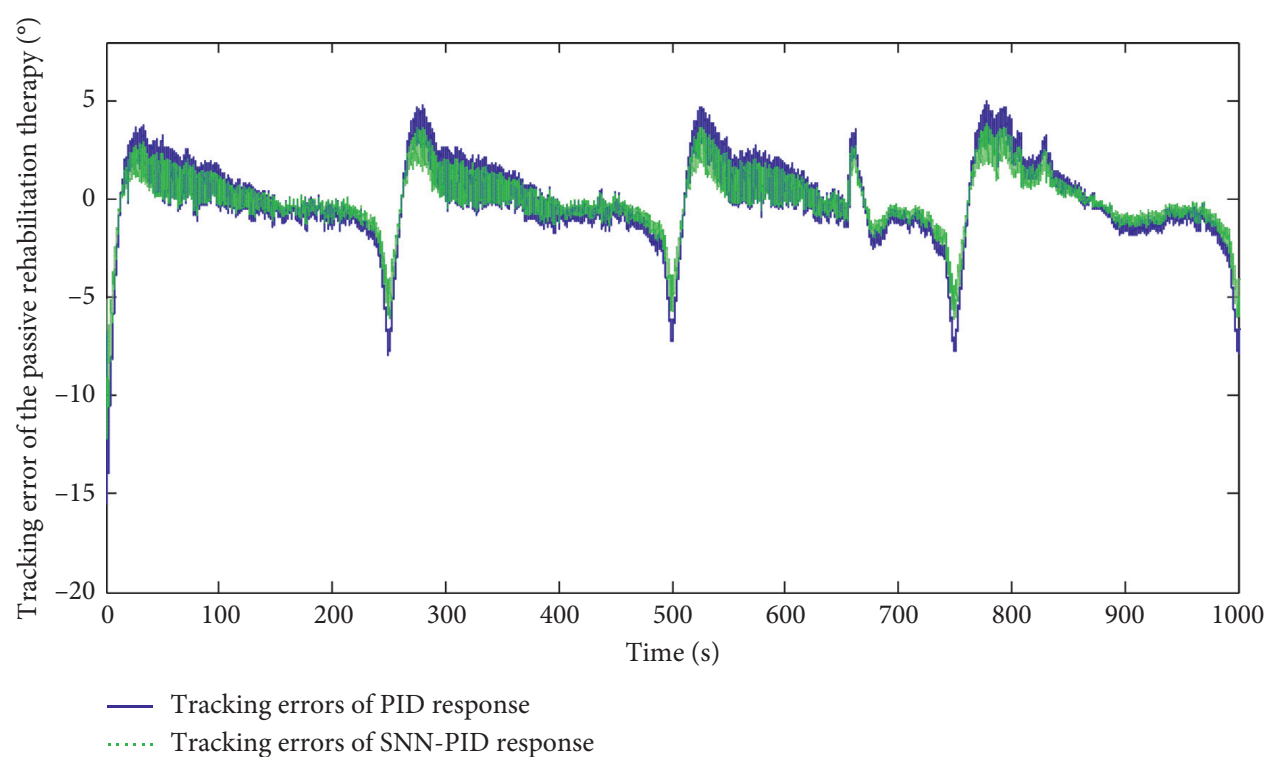

FIGURE 12: Tracking errors of passive rehabilitation therapy experiment under a half sinusoidal wave excitation of $0.004 \mathrm{~Hz}$ with amplitude $50^{\circ}$. 


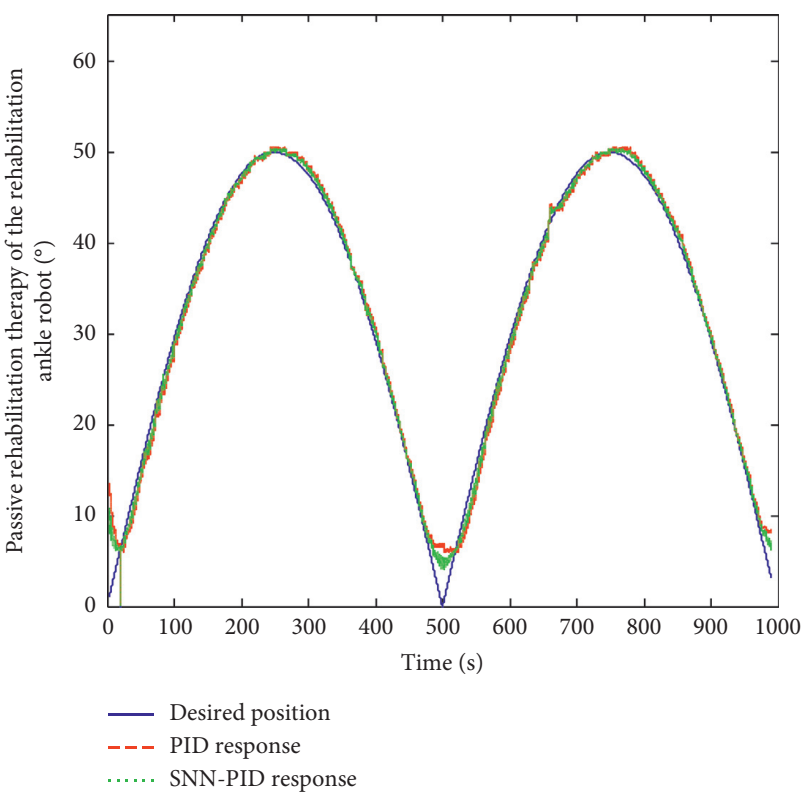

(a)

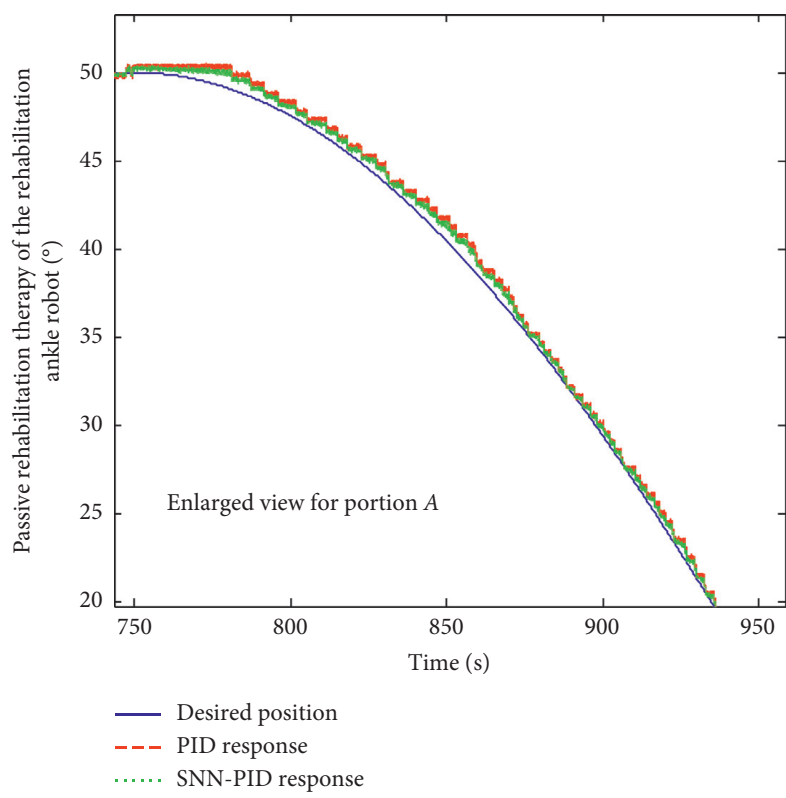

(b)

Figure 13: Passive rehabilitation therapy experiment under a half sinusoidal wave excitation of $0.002 \mathrm{~Hz}$ with amplitude $50^{\circ}$ : (a) tracking performance; (b) enlarged view for portion $\mathrm{A}$ in (a).

curves of the two controllers demonstrates better capacity of single neuron tuned PID algorithm in overcoming nonlinearities and disturbances of the robot.

\section{Conclusions}

Compliant ankle rehabilitation robot in this research is realized by employing pneumatic muscle actuators as the power source. A pair of PMAs is arranged in antagonistic form and provides driving torque through crank-slider mechanism. Prototype is manufactured and assembled. A single neuron tuned PID controller with torque safety switch is designed for the robot. Passive rehabilitation experiments are conducted and effectiveness of the adaptive controller is validated. Conclusion of experiments demonstrates that the devised controlling algorithm can improve accuracy of position tracking of the robot.

\section{Data Availability}

The datasets used or analyzed during the current study are available from the corresponding author on reasonable request.

\section{Conflicts of Interest}

The authors declare that there are no conflicts of interest regarding the publication of this paper.

\section{Acknowledgments}

This work was supported in part by the National Key R\&D Program of China under Grants 2019YFC1511400, 2019YFC1511401, and 2019YFC1511402, Pre-research
Union Fund of China Ministry of Education \&\& PLA Equipment Development Department under Grant 6141A02033616, Fundamental Research Funds for the Central Universities under Grant B200202223, Strategic Rocket Innovation Fund of National Defense Science and Technology Industry under Grant Y18143, Natural Science Foundation of Jiangsu Province under Grant BK20170301, and Opening Fund of Jiangsu Key Laboratory of Special Robot Technology under Grant 2017JSJQR01.

\section{References}

[1] S. Okada, T. Sakaki, R. Hirata, Y. Okajima, S. Uchida, and Y. Tomita, "TEM: a therapeutic exercise machine for the lower extremities of spastic patients," Advanced Robotics, vol. 14, no. 7, pp. 597-606, 2001.

[2] D. Bradley, C. Acosta-Marquez, M. Hawley, S. Brownsell, P. Enderby, and S. Mawson, "NeXOS-the design, development and evaluation of a rehabilitation system for the lower limbs," Mechatronics, vol. 19, no. 2, pp. 247-257, 2009.

[3] M. Mawson, H. A. M. Rittenhouse, and H. A. Abdullah, "Design issues for therapeutic robot systems: results from a survey of physiotherapists," Journal of Intelligent and Robotic Systems, vol. 42, no. 3, pp. 239-252, 2005.

[4] P. Métrailler, V. Blanchard, I. Perrin et al., "Improvement of rehabilitation possibilities with the motion maker TM," in Proceedings of the IEEE/RAS-EMBS International Conference on Biomedical Robotics \& Biomechatronics, 2006. BioRob 2006, Pisa, Italy, February 2006.

[5] M. L. Aisen, H. I. Krebs, N. F. Hogan, and B. T. Volpe, "The effect of robot-assisted therapy and rehabilitative training on motor recovery following stroke," Archives of Neurology, vol. 54, no. 4, pp. 443-446, 1997.

[6] C. McDowell, C. Chen, N. Wang, Z. Ju, J. Fu, and M. Wang, "Biologically inspired motion modeling and neural control for robot learning from demonstrations," IEEE Transactions on 
Cognitive and Developmental Systems, vol. 11, no. 2, pp. 281-291, 2019.

[7] T. Tsuji and Y. Tanaka, "Bio-mimetic impedance control of robotic manipulator for dynamic contact tasks," Robotics and Autonomous Systems, vol. 56, no. 4, pp. 306-316, 2008.

[8] C. P. Bechlioulis and G. A. Rovithakis, "Adaptive control with guaranteed transient and steady state tracking error bounds for strict feedback systems," Automatica, vol. 45, no. 2, pp. 532-538, 2009.

[9] C. P. Bechlioulis and G. A. Rovithakis, "Robust adaptive control of feedback linearizable MIMO nonlinear systems with prescribed performance," IEEE Transactions on Automatic Control, vol. 53, no. 9, pp. 2090-2099, 2008.

[10] R. Riener, S. L. Lunenburger, S. Jezernik, M. Anderschitz, G. Colombo, and V. Dietz, "Patient-cooperative strategies for robot-aided treadmill training: first experimental results," IEEE Transactions on Neural Systems and Rehabilitation Engineering, vol. 13, no. 3, pp. 380-394, 2005.

[11] C. Dietz, J. Luo, C. Liu, M. Li, and S.-L. Dai, "Haptics electromyography perception and learning enhanced intelligence for teleoperated robot," IEEE Transactions on Automation Science and Engineering, vol. 16, no. 4, pp. 1512-1521, 2019.

[12] U. Demir, S. Kocaoğlu, and E. Akdogan, "Human impedance parameter estimation using artificial neural network for modelling physiotherapist motion," Biocybernetics and Biomedical Engineering, vol. 36, no. 2, Article ID S0208521616300031, 2016.

[13] S. Mohan, J. K. Mohanta, S. Kurtenbach, J. Paris, B. Corves, and M. Huesing, "Design, development and control of a 2PRP-2PPR planar parallel manipulator for lower limb rehabilitation therapies," Mechanism and Machine Theory, vol. 112, pp. 272-294, 2017.

[14] J. Wu, J. Gao, R. Song, R. Li, Y. Li, and L. Jiang, "The design and control of a 3DOF lower limb rehabilitation robot," Mechatronics, vol. 33, pp. 13-22, 2016.

[15] S. K. Banala, S. H. Kim, S. K. Agrawal, and J. P. Scholz, "Robot assisted gait training with active leg exoskeleton (ALEX)," IEEE Transactions on Neural Systems \& Rehabilitation Engineering, vol. 17, no. 1, pp. 2-8, 2009.

[16] C. Yang, J. Luo, Y. Pan, Z. Liu, and C.-Y. Su, "Personalized variable gain control with tremor attenuation for robot teleoperation," IEEE Transactions on Systems, Man, and Cybernetics: Systems, vol. 48, no. 10, pp. 1759-1770, 2017.

[17] R. Pérez-Rodríguez, C. Rodríguez, Ú. Costa et al., “Anticipatory assistance-as-needed control algorithm for a multijoint upper limb robotic orthosis in physical neurorehabilitation," Expert Systems with Applications, vol. 41, no. 8, pp. 39223934, 2014.

[18] S. Hussain, S. Q. Xie, and P. K. Jamwal, "Adaptive impedance control of a robotic orthosis for gait rehabilitation," IEEE Transactions on Cybernetics, vol. 43, no. 3, pp. 1025-1034, 2013.

[19] W. Meng, Q. Liu, Z. Zhou, and Q. Ai, "Active interaction control applied to a lower limb rehabilitation robot by using EMG recognition and impedance model," Industrial Robot: An International Journal, vol. 41, no. 5, pp. 465-479, 2014.

[20] K. Liu, J.-C. Zhao, E.-G. Cao, and X. Han, "Control methods for standing-up rehabilitation robot based on estimation and analysis of lower limb kinetics," Jilin Daxue Xuebao (Gongxueban)/Journal of Jilin University (Engineering and Technology Edition), vol. 45, no. 3, pp. 837-843, 2015.

[21] C. Yang, C. Chen, W. He, R. Cui, and Z. Li, "Robot learning system based on adaptive neural control and dynamic movement primitives," IEEE Transactions on Neural Networks and Learning Systems, vol. 30, no. 3, pp. 777-787, 2019.
[22] F. Yongfei, H. Wang, H. Yan, X. Wang, Z. Jin, and L. Vladareanu, "Research on safety and compliance of a new lower limb rehabilitation robot," Journal of Healthcare Engineering, vol. 2017, Article ID 1523068, 11 pages, 2017.

[23] W. Meng, Q. Liu, Z. Zhou, Q. Ai, B. Sheng, and S. Xie, "Recent development of mechanisms and control strategies for robotassisted lower limb rehabilitation," Mechatronics, vol. 31, pp. 132-145, 2015.

[24] J. Yoon, B. Novandy, C.-H. Yoon, and K.-J. Park, "A 6-DOF gait rehabilitation robot with upper and lower limb connections that allows walking velocity updates on various terrains," IEEE/ASME Transactions on Mechatronics, vol. 15, no. 2, pp. 201-215, 2010.

[25] S. M. M. Rahman and R. Ikeura, "A novel variable impedance compact compliant ankle robot for overground gait rehabilitation and assistance," Procedia Engineering, vol. 41, pp. 522-531, 2012.

[26] H. Stefan, J. Mehrholz, and C. Werner, "Robot-assisted upper and lower limb rehabilitation after stroke: walking and arm/ hand function," Deutsches Rzteblatt International, vol. 105, no. 18, pp. 330-336, 2008.

[27] X. H. Shi, H. B. Wang, L. Yuan, Z. Xu, H. W. Zhen, and Z. G. Hou, "Design and analysis of a lower limb rehabilitation robot," Advanced Materials Research, vol. 490-495, pp. 2236-2240, 2012.

[28] N. Wang, C. Chen, and A. Di Nuovo, "A framework of hybrid force/motion skills learning for robots," IEEE Transactions on Cognitive and Developmental Systems, p. 1, 2020, In press.

[29] Y. Pei, Y. Kim, G. Obinata, K. Hase, and D. Stefanov, "Trajectory planning of a robot for lower limb rehabilitation," in Proceedings of the 2011 Annual International Conference of the IEEE Engineering in Medicine and Biology Society, pp. 12591263, Boston, MA, USA, August 2011.

[30] M. M. Fateh and V. Khoshdel, "Voltage-based adaptive impedance force control for a lower-limb rehabilitation robot," Advanced Robotics, vol. 29, no. 15, pp. 961-971, 2015. 